\title{
Structural and evolutionary divergence of aquaporins in parasites (Review)
}

\author{
ZI-XIN NI ${ }^{1}$, JIAN-MIN CUI ${ }^{2}$, NIAN-ZHANG ZHANG ${ }^{2}$ and BAO-QUAN FU ${ }^{2,3}$ \\ ${ }^{1}$ College of Veterinary Medicine, China Agricultural University, Beijing 100083; \\ ${ }^{2}$ State Key Laboratory of Veterinary Etiological Biology, Key Laboratory of Veterinary Parasitology of Gansu, \\ Lanzhou Veterinary Research Institute, Chinese Academy of Agricultural Sciences, \\ Lanzhou, Gansu 730046; ${ }^{3}$ Jiangsu Co-innovation Center for Prevention and Control of \\ Important Animal Infectious Disease, Yangzhou, Jiangsu 225009, P.R. China
}

Received March 21, 2016; Accepted February 28, 2017

DOI: $10.3892 / \mathrm{mmr} .2017 .6505$

\begin{abstract}
Aquaporins are ubiquitous proteins that belong to the major intrinsic protein family. Previous studies have indicated that aquaporins are involved in multiple physiological processes in parasites, such as nutrient absorption and end product efflux, and thus, would be promising pharmacological agents in the fight against parasite infection. In the present paper, the authors analyzed the evolutionary relationship of parasitic aquaporins by re-constructing of a phylogenic tree using neighbor-joining and maximum likelihood methods. In addition, the authors discussed the variation of the conserved functional sites impacting on the transportation of water molecules. The protein was concluded to be a potential drug target in parasites.
\end{abstract}

\section{Contents}

1. Introduction

2. Re-construction of phylogenetic tree based on aquaporin sequences

3. The variation of restrictions in aquaporin proteins from parasites

4. The variation of the NPA motifs in aquaporin of parasites

5. Potential drug targets in parasites: Aquaporins

6. Conclusions and perspectives

Correspondence to: Professor Bao-Quan Fu, State Key Laboratory of Veterinary Etiological Biology, Key Laboratory of Veterinary Parasitology of Gansu, Lanzhou Veterinary Research Institute, Chinese Academy of Agricultural Sciences, 1 Xujiaping Street, Lanzhou, Gansu 730046, P.R. China

E-mail: fubaoquan@caas.cn

Key words: aquaporin, evolution, genetic variation, drug targets

\section{Introduction}

Parasites can be divided within a diverse phylum composed of protozoan and helminthes, and this phylum contains numerous species that can infect humans and animals. Some parasitic species can even infect plants, and have been affecting humans for many years (1). Emerging parasitic diseases in humans and animals, such as malaria, toxoplasmosis, leishmaniasis, schistosomiasis, echinococcosis, trichinellosis and cysticercosis, are global problems, which can lead to serious nutritional deficiencies, reduce animal productivity, effect acute tissue damage or even death (2-5).

Considering that parasites have diverse and complex biological and ecological lifestyles, this raises questions as to how can they maintain their fluid homeostasis under different osmotic stress in vivo and in vitro, and also how parasites regulate rapid water transport in moist and dry environments. What is interesting about these parasites are the channels and transporters (also known as permeases) in membranes with essential biological functions involving in facilitating water transport and osmoregulation, nutrient uptake, cytotoxic release and host cellular adhesion $(6,7)$.

A significant discovery concerning channels in cell membranes awarded the Nobel Prize in Chemistry in 2003 to Peter Agre, who was famed for identifying the functions of aquaporins (AQPs). In the present paper, the authors review the amino acid residue divergence and analyze the evolutionary relationship of parasite aquaporins, and further discuss the possibility of developmental drug design against helminths based on the protein.

AQPs belong to the major intrinsic protein (MIP) family and exist in almost all living organisms including animals, plants, bacteria and viruses (8). The aquaporin family is abundant in the plasma membrane (9). Proteomics analyses indicate that aquaporins are the most plentiful proteins within the biological tegument surface of Schistosoma mansoni (10). The common structure of AQPs consists of $6 \alpha$-helical transmembrane domains (TM) and five connecting loops (11). The $\mathrm{N}$ - and $\mathrm{C}$-terminal regions of AQPs, as well as B and D loops, are within the cytoplasm.

The advanced structure of AQP proteins is often compared to an hourglass, whereby the center of the pore has unique 
Asn-Pro-Ala (NPA) sequence motifs in loops B and E; this is where an isolated water molecule will transiently form hydrogen bonds with two conserved Asn residues (12). The narrowest restricted site is located at the entrance of the pore mouth with a diameter of $2.8 \AA$, which may interact with passing solutes, bound by aromatic amino acids and a widely conserved aromatic arginine (ar/R) filter $(12,13)$.

\section{Re-construction of phylogenetic tree based on aquaporin sequences}

To investigate the evolutionary relationship of MIP family proteins in parasites, the deduced polypeptides were investigated by ClustalW multiple sequence alignment software and the phylogenetic tree of parasites was re-constructed using the neighbor-joining (NJ) and maximum likelihood (ML) methods, based on the AQP sequences (Fig. 1). A total of 44 AQP sequences were selected. The most outstanding phylogenetic pattern is divided into two distinct clusters that are orthodox aquaporins and aquaglyceroporins (GLPs). The orthodox aquaporin, also named classical aquaporin, only allow the passage of water, while the aquaglyceroporins can also transport uncharged molecules like polyols, urea and metalloid besides water (14). In the phylogenic tree, a subcellular-aquaporin group is also formed as an independent cluster, due to the unusual conserved NPA motifs $(15,16)$.

Unlike the aquaporins in plants and mammalian animals, the protein in parasites seems to have fewer isoforms (17), and thus, the few channels tend to be multifunctional. The assembled functions in a single protein are not only due to the simple physiological structures of parasites, but also due to the probability of a self-protection strategy that parasites use to reduce excessive antigen exposure to the host-parasite interface; this avoids activating the host immune response system.

As revealed from the phylogenetic tree in the present study, GLP group can be further divided into four major GLPs sub-groups: i) Protozoon GLPs+Homo sapiens $(\mathrm{Hs}) \mathrm{AQP}_{7}$ and Escherichia coli (Ec) GlpF, ii) nematode GLPs + HsAQP ${ }_{3,9,10}$, iii) trematode GLPs, and iv) cestode GLPs. These parasites possess at least one multi-functional aquaporin. It is noteworthy that both $\mathrm{FgAQP}_{1}$ and $\mathrm{FgAQP}$ were integrated into in a single branch belonging to subcellular-aquaporin group, both of which contain a mutated TAA motif in the B loop (18). FgAQPs are primarily expressed in tegumental cells and the linings of ovary and testes (18). Increased water permeability observed in Xenopus oocytes, but a failure to permeate glycerol and urea (18) suggests that FgAQPs may be more likely to involve osmoregulation in Fasciola instead of transporting solutes.

Similarly interesting is the distribution of Toxoplasma gondii (Tg)AQP. In the phylogenetic tree, TgAQP belongs to the water specific group that presents $47 \%$ similarity to plant tonoplast intrinsic proteins, which is a water specific channel in theory. Pavlovic-Djuranovic, Schultz and Beitz (19) reported that TgAQP is a bifunctional channel, permeating both water and glycerol, which suggests that TgAQP has obtained solute permeability following gene transfer from plant to an ancestor of Toxoplasma.

\section{The variation of restrictions in aquaporin proteins from parasites}

Aquaporin integrates into a homotetramer in the plasma membrane and each monomer is an independent functional pore (20). The central pore formed by four monomers is said to be a gas channel for $\mathrm{CO}_{2}$ and $\mathrm{NO}(21)$. Arg ( $\mathrm{ar} / \mathrm{R}$ ) constriction and NPA motifs are two major constriction regions that are responsible for aquaporin selectivity. The ar/ $\mathrm{R}$ constriction region is the narrowest part of the water channel, and the residues are Phe56, His180, Cys189 and Arg195 in human AQP1 and Trp48, Gly191, Phe200 and Arg206 in E. coli GlpF. The substitution of His180, Cys189 in GlpF by Gly191 and Phe200 may result in the changed size and the increased hydrophobicity, which allow a larger molecule, such as glycerol, to pass through $(22,23)$.

Here, the ClustalW analysis demonstrated that the most remarkable point lies in the highly conserved arginine residue (Figs. 2 and 3) following the second NPA box, except in 4 AQP sequences (TbAQP, TgAQP, FgAQP ${ }_{1,2}, \mathrm{HsAQP}_{11,12}$ ). Arginine is a polar molecule with positive charges, and serves a vital role in the transport selectivity and expulsion of proton (23). The removal of the positive charge AQP1-R195V or AQP1-H180A/R195V would result in proton leak (23). In TgAQP, the Arg204 residue mutates into valine, a similar mutation to the plant TIP subfamily (19). The unusual selectivity filter replaces Arg264 with Leu264 in $\mathrm{TbAQP}_{2}$ and displays the ability to allow the passage of large molecules like melarsoprol and pentamidine through the channel (24). As a result, $\mathrm{TbAQP}_{2}$ may transport larger solutes compared with $\mathrm{TbAQP}_{3}$, and may be responsible for it susceptibility of melarsoprol and pentamidine (24). In addition, the cysteine residue serves a significant role in the ar/R restriction. Cys in AQPs is a vital residue reacting to mercurial compounds. $\mathrm{HsAQP}_{1}(\mathrm{C} 189)$ and $\mathrm{HsAQP}_{9}(\mathrm{C} 213)$ can be inhibited by mercuric chloride $(25,26)$. It can be suggested that cysteines at position 204, 203 and 220 in $\mathrm{FgAQP}_{1}, \mathrm{FgAQP}_{2}$ and $\mathrm{OvAQP}_{3}$, respectively, may also be mercury-sensitive sites and could be blocked by mercury ions.

The sequences used in the current work indicated that the functional residues were corresponding to the Froger's prediction method (27) at $\mathrm{P}_{1-5}$ positions, although limited aquaporin sequences have been used (40 aquaporin sequences in total, 14 for GlpF cluster, 26 for AQP cluster). In addition, the authors previously identified some more conserved residues (data not shown), but their roles are still unclear and need to be confirmed later in detail.

\section{The variation of the NPA motifs in aquaporin of parasites}

NPA motifs are characteristics of MIP family members, comprising Asn-Pro-Ala (11). The Asn residue can be considered as the secondary proton filter pointing towards the inner channel, of which the side chains serve as hydrogen-bond donors, forming new hydrogen bonds with travelling water molecules (28).

As presented in the aligned sequences, NPA boxes (Figs. 2 and 3) are highly conserved in MIP family members, despite of a few variations. The variations were primarily at the second or third position in the first NPA motif, except in $\mathrm{FgAQP}_{1-2}$. The NPA motif in FgAQP ${ }_{1-2}$ comprises TAA motif 


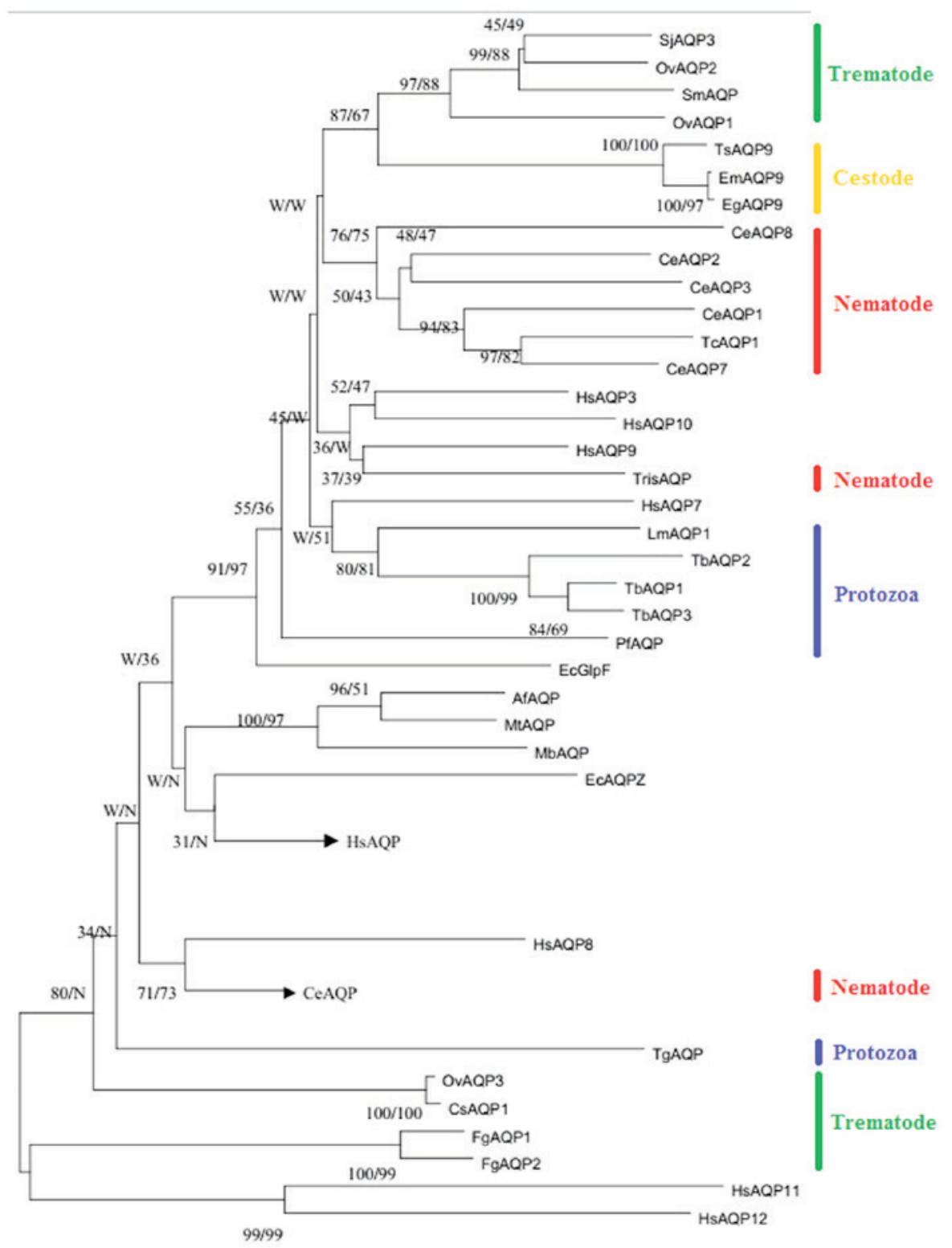

Figure 1. The phylogenetic tree re-constructed using NJ and ML methods to elucidate the evolutionary relationship between parasitic aquaporin sequences. The accession numbers for each sequence are as follows: PfAQP (AJ413249); LmAQP1 (AY567835); TbAQP1-3 (AJ697889, AJ697890, AJ697891); TgAQP (Toxoplasma gondii); SjAQP3 (CPRT0000005211); SmAQP (EU780065); FgAQP1-2 (HM748645, HM748644); TcAQP1 (AF067963); CeAQP1-8 (CCD66276, CAA84633, CAA22259, CAA94770, CAA94903, CCF23336, CCD66489, CCD68566); HsAQP1-12 (NP_932,766, AAB31999, CAG46822, CAG46819, NP 0,01643, CAI13303, AAH40630, CAG46824, CAH70483, Q8NBQ7, Q8IXF9); TrisAQP (unclear); EcGlpF (CDZ22687); EcAQPZ (AAC43518); EmAQP9 (CDS35949); EgAQP9 (CDS22736); TsAQP9 (000,547100); AfAQP (NP_07,0255); MbAQP (ZP_00,077803); MtAQP (AB055880); OvAQP1-3 (KF697690, KF697691, KM359766); CsAQP1 (GAA33659). The numbers indicate bootstrap values resulting from different analyses in the order NJ/ML. NJ, neighbor-joining; ML, maximum likelihood; AQP, aquaporin; Pf, Plasmodium falciparum; Lm, Leishmania major; Tb, Trypanosoma brucei; $\mathrm{Tg}$, Toxoplasma gondii; Sj, Schistosoma japonicum; Sm, Schistosoma mansoni; Fg, Fasciola gigantica; Tc, Toxocara canis; Ce, Caenorhabditis elegans; Hs, Homo sapiens; Tris, Trichinella spiralis; Ec, Escherichia coli; Em, Echinococcus multilocularis; Eg, Echinococcus granulosus; Ts, Taenia solium; Af, archaeoglobus fulgidus; $\mathrm{Mb}$, Methanosarcina barkeri; $\mathrm{Mt}$, Methanothermobacter thermautotrophicus; Ov, Opisthorchis viverrini; Cs, Clonorchis sinensis.

(Thr-Ala-Ala), and the similar formation is demonstrated in the Burkholderia coenocepacia SPA at a corresponding position (29). In fewer cases, including PfAQP, TbAQP $\mathrm{CeAQP}_{5}$ and $\mathrm{HsAQP}_{7}$, both of the two NPA motifs become distinct in a single aquaporin sequence $(30,31)$. These mutations alter the bulk and polarity of amino acid residues, which leads to the changed hydrophilic property and the physiological roles. Replacement of Thr by Asn at the first TAA motif of FgAQP1 made its water flux increase two-fold when expressed in Xenopus laevis oocytes, however, this alteration does not alter the impermeability of urea and glycerol (18). In
Trypanosoma brucei, TbAQP2 contains two non-canonical NSA/NPS motifs that are scarcely expressed. Perhaps the protein is an organelle aquaporin and is expressed only under given stress conditions (30).

The altered motifs, however, do not always alter its transport properties (31). In Plasmodium falciparum, the classical NPA sequences were substituted with the uncommon NLA and NPS sequences in B and E loops, respectively. After the divergent motifs are converted to canonical NPA motifs, the swelling rates of the mutative protein to water and glycerol are equal with wild type PfAQP (31). An identical variation 


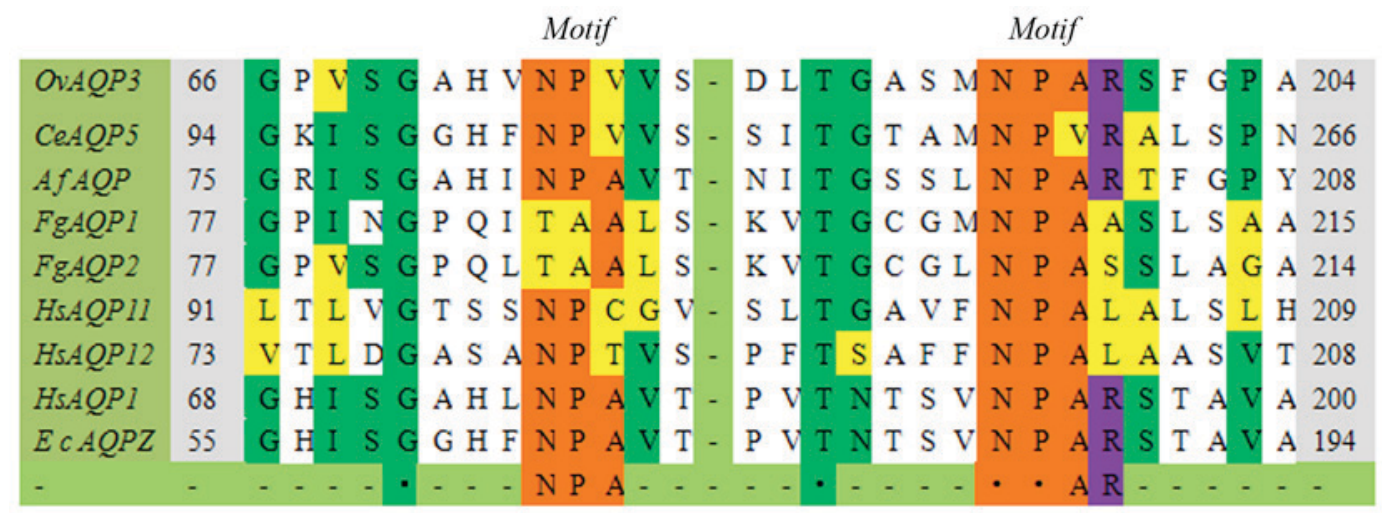

Figure 2. Partial aquaporin sequences (including FgAQP1-2, HsAQP11-12) were aligned to display the variations at NPA motifs and Arg position. No variation. Fg, Fasciola gigantica; AQP, aquaporin; Hs, Homo sapiens; Ov, Opisthorchis viverrini; Ce, Caenorhabditis elegans; Af, archaeoglobus fulgidus; Ec, Escherichia coli.

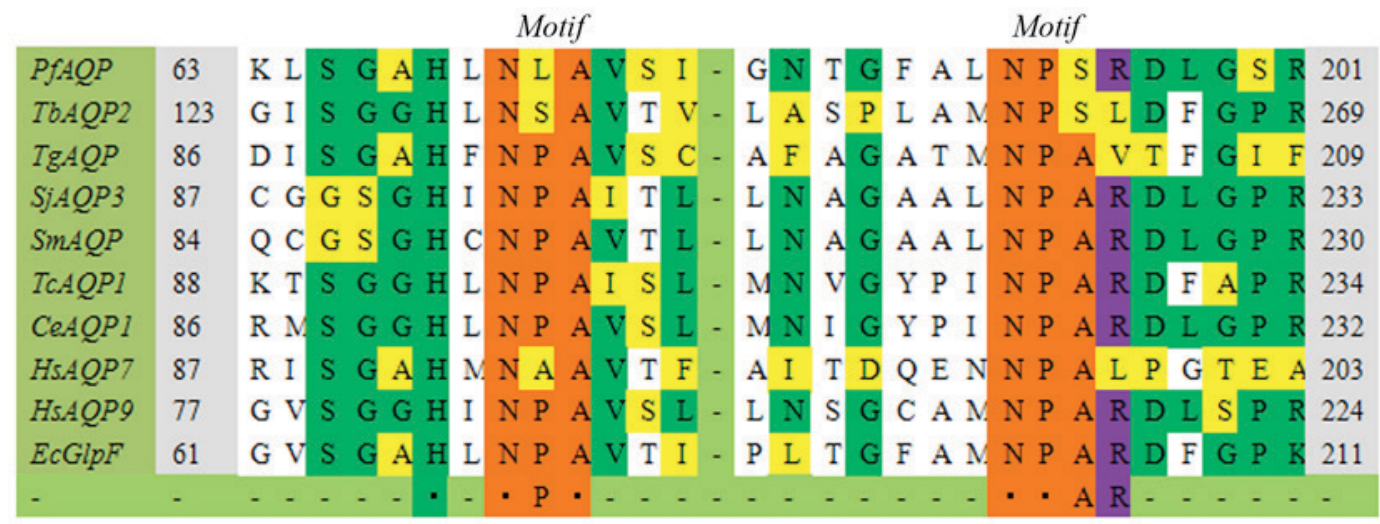

Figure 3. Partial GlpFs sequences were aligned to display the variations at NPA motifs and Arg position. "no variation. Pf, Plasmodium falciparum; Tb, Trypanosoma brucei; Tg, Toxoplasma gondii; $\mathrm{Sj}$, Schistosoma japonicum; $\mathrm{Sm}$, Schistosoma mansoni; Tc, Toxocara canis; Ce, Caenorhabditis elegans; $\mathrm{Hs}$, Homo sapiens; Ec, Escherichia coli.

at the first NPA (changed into NPV) motif was identified in $\mathrm{OvAQP}_{3}$ and $\mathrm{CsAQP}_{1}$ (32). The $\mathrm{OvAQP}_{3}$ was predicted to be a similar advanced structure to mammalian $\mathrm{AQP}_{1}$, the specific water channel. However, $\mathrm{OvAQP}_{3}$ can transport glycerol and urea, but not only water molecules, which suggests that sequence-based aquaporin classification do not completely reveal its real function in parasites (32).

\section{Potential drug targets in parasites: Aquaporins}

Aquaporins can regulate multiple significant physiological roles in living organisms, which means that they are regarded as prime candidates for pharmacological intervention in human diseases (33). The numbers of aquaporin-encoding genes are varied in parasites, for instance, there are five genes in Leishmania major and one gene in P. falciparum $(31,34)$. AQPs are desired as novel targets for antiparasitic drugs $(35,36)$.

Aquaglyceroporins isolated from Leishmania major $(\mathrm{Lm})$ AQP1 and Schistosoma mansoni (Sm)AQP are physiological water channels. They can act as conduit for parasite-killing drugs and increase the accumulation of metalloids, such as $\mathrm{As}(\mathrm{III})$ and $\mathrm{Sb}(\mathrm{III})$ in L. major and Potassium Antimonyl Tartrate in S. mansoni $(36,37)$. A previous study demonstrated that, following silencing SmAQP using specific
siRNA, the treated Schistosomula exhibits more resistance to PAT (36). Proteomic analysis of the Schistosome tegument membranes presented a single AQP homologue (38), which exhibits 31-36\% sequence identity with human AQP 3,7,9 and 10. In addition, SmAQP may also act as a channel for glycolytic end-products to avoid the accumulation of toxins (39). The Arg residue located inside the vestibule of the channel may also severely affect the metalloid sensitivity (40). For instance, alteration of Arg230 in LmAQP1 to Ala or Lys presented a negligible low level transport of metalloid in cells, and therefore appeared more resistant to As(III) and $\mathrm{Sb}$ (III) than cells expressing wild type LmAQP1 (41). The side chain of the Ala residue at the position of 163 in LmAQP1 may serve a role in drug resistance because of a steric hindrance effect (42). The Thr164 substituted by Cys in LmAQP1 altered the mercurial sensitivity that can be blocked by $\mathrm{HgCl}_{2}$ (42). These studies suggested that aquaporin in parasites possess transport property for drugs and, therefore, may have potential access to be a viable candidate for therapeutic targets.

\section{Conclusions and perspectives}

Since the discovery of the first aquaporin, the protein has attracted considerable interests in the development of new 
pharmacological agents for the treatment of parasitological diseases, due to their potential structure and functions. In general, aquaporins are conserved between species during the evolutionary process, especially at the transmembrane regions and functional sites (e.g., NPA and ar/R). In parasitic aquaporins, variations seem to have differential effects for different aquaporin, but not always altering the selectivity of the aquaporin. As the common component of the parasite-host interface, aquaporins possess great advantages of being new chemotherapeutic targets. However, few helminthic aquaporins have been well studied.

Some compounds, such as mercuric chloride, can act as aquaporin inhibitors, interdicting the ability of the protein to transport solutes (43). Some aquaporin modulators are promising agents for the treatment of human disorders (33). Furthermore, the elucidation of 3D structures and more crystallographic information of aquaporins will increase our understanding of drug design. However, much requires elucidation in order to develop the potential applications of parasitic aquaporins. The development of mammalian aquaporins would be provided references for the studies on parasitic aquaporins. In summary, aquaporins are an ancient protein with the ability of transportation of water molecules and nutrients, osmoregulation, invasion and drug resistance. More studies on parasitic aquaporins are required.

\section{Acknowledgements}

Project support was provided by the Science Fund for Creative Research Groups of Gansu Province (grant no. 1210RJIA006).

\section{References}

1. Mehlhorn H: Encyclopedia of parasitology I. 3rd edition. Springer, 2008.

2. Ramasamy R: Zoonotic malaria-global overview and research and policy needs. Front Public Health 2: 123, 2014.

3. Halonen SK and Weiss LM: Toxoplasmosis. Handb Clin Neurol 114: 125-145, 2013.

4. Pace D: Leishmaniasis. J Infect 69 (Suppl 1): S10-S18, 2014.

5. Robinson MW and Dalton JP: Zoonotic helminth infections with particular emphasis on fasciolosis and other trematodiases. Philos Trans R Soc Lond B Biol Sci 364: 2763-2776, 2009.

6. Kirk K: Channels and transporters as drug targets in the Plasmodium-infected erythrocyte. Acta Trop 89: 285-298, 2004

7. Sibley LD: How apicomplexan parasites move in and out of cells. Curr Opin Biotechnol 21: 592-598, 2010.

8. Gomes D, Agasse A, Thiébaud P, Delrot S, Gerós H and Chaumont F: Aquaporins are multifunctional water and solute transporters highly divergent in living organisms. Biochim Biophys Acta 1788: 1213-1228, 2009.

9. Shiels A: Focus on molecules: Major intrinsic protein. Exp Eye Res 101: 107-108, 2012.

10. Castro-Borges W, Simpson DM, Dowle A, Curwen RS, Thomas-Oates J, Beynon RJ and Wilson RA: Abundance of tegument surface proteins in the human blood fluke Schistosoma mansoni determined by QconCAT proteomics. J Proteomics 74: 1519-1533, 2011.

11. Fujiyoshi Y, Mitsuoka K, de Groot BL, Philippsen A, Grubmüller H, Agre P and Engel A: Structure and function of water channels. Curr Opin Struct Biol 12: 509-515, 2002.

12. Sui H, Han BG, Lee JK, Walian P and Jap BK: Structural basis of water-specific transport through the AQP1 water channel. Nature 414: 872-878, 2001.

13. Campbell EM, Ball A, Hoppler S and Bowman AS: Invertebrate aquaporin: A review. J Comp Physiol B 178: 935-955, 2008.

14. Liu Z, Shen J, Carbrey JM, Mukhopadhyay R, Agre P and Rosen BP: Arsenite transport by mammalian aquaglyceroporins AQP7 and AQP9. Proc Natl Acad Sci USA 99: 6053-6058, 2002.
15. Yakata K, Hiroaki Y, Ishibashi K, Sohara E, Sasaki S, Mitsuoka K and Fujiyoshi Y: Aquaporin-11 containing a divergent NPA motif has normal water channel activity. Biochim Biophys Acta 1768: 688-693, 2006

16. Itoh T, Rai T, Kuwahara M, Ko SB, Uchida S, Sasaki S and Ishibashi K: Identification of a novel aquaporin, AQP12, expressed in pancreatic acinar cells. Biochem Biophys Res Commun 330: 832-838, 2005.

17. Yue C, Cao H, Wang L, Zhou Y,Hao X,Zeng J, Wang X and Yang Y: Molecular cloning and expression analysis of tea plant aquaporin (AQP) gene family. Plant Physiol Biochem 83: 65-76, 2014.

18. Geadkaew A, von Bülow J, Beitz E, Grams SV, Viyanant V and Grams R: Functional analysis of novel aquaporins from Fasciola gigantica. Mol Biochem Parasitol 175: 144-153, 2011.

19. Pavlovic-Djuranovic S, Schultz JE and Beitz E: A single aquaporin gene encodes a water/glycerol/urea facilitator in Toxoplasma gondii with similarity to plant tonoplast intrinsic proteins. FEBS Lett 555: 500-504, 2003.

20. Murata K, Mitsuoka K, Hirai T, Walz T, Agre P, Heymann JB, Engel A and Fujiyoshi Y: Structural determinants of water permeation through aquaporin-1. Nature 407: 599-605, 2000.

21. Herrera M and Garvin JL: Aquaporins as gas channels. Pflugers Arch 462: 623-630, 2011.

22. Stroud RM, Savage D, Miercke LJ, Lee JK, Khademi S and Harries W: Selectivity and conductance among the glycerol and water conducting aquaporin family of channels. FEBS Lett 555: 79-84, 2003.

23. Beitz E, Wu B, Holm LM, Schultz JE and Zeuthen T: Point mutations in the aromatic/arginine region in aquaporin 1 allow passage of urea, glycerol, ammonia, and protons. Proc Natl Acad Sci USA 103: 269-274, 2006.

24. Baker N, Glover L, Munday JC, Aguinaga Andrés D, Barrett MP, de Koning HP and Horn D: Aquaglyceroporin 2 controls susceptibility to melarsoprol and pentamidine in African trypanosomes. Proc Natl Acad Sci USA 109: 10996-1001, 2012.

25. Preston GM, Jung JS, Guggino WB and Agre P: The mercury-sensitive residue at cysteine 189 in the CHIP28 water channel. J Biol Chem 268: 17-20, 1993.

26. Ishibashi K, Kuwahara M, Gu Y, Tanaka Y, Marumo F and Sasaki S: Cloning and functional expression of a new aquaporin (AQP9) abundantly expressed in the peripheral leukocytes permeable to water and urea, but not to glycerol. Biochem Biophys Res Commun 244: 268-274, 1998.

27. Froger A, Tallur B, Thomas D and Delamarche C: Prediction of functional residues in water channels and related proteins. Protein Sci 7: 1458-1468, 1998.

28. Chakrabarti N, Tajkhorshid E, Roux B and Pomès R: Molecular basis of proton blockage in aquaporins. Structure 12: 65-74, 2004.

29. Wree D, Wu B, Zeuthen T and Beitz E: Requirement for asparagine in the aquaporin NPA sequence signature motifs for cation exclusion. FEBS J 278: 740-748, 2011.

30. Uzcategui NL, Szallies A, Pavlovic-Djuranovic S, Palmada M, Figarella K, Boehmer C, Lang F, Beitz E and Duszenko M: Cloning, heterologous expression, and characterization of three aquaglyceroporins from Trypanosoma brucei. J Biol Chem 279: 42669-42676, 2004

31. Hansen M, Kun JF, Schultz JE and Beitz E: A Single, bi-functional aquaglyceroporin in blood-stage Plasmodium falciparum malaria parasites. J Biol Chem 277: 4874-4882, 2002.

32. Geadkaew A, von Bülow J, Beitz E, Tesana S, Vichasri Grams S and Grams R: Bi-functionality of Opisthorchis viverrini aquaporins. Biochimie 108: 149-159, 2015.

33. Huber VJ, Tsujita M and Nakada T: Aquaporins in drug discovery and pharmacotherapy. Mol Aspects Med 33: 691-703, 2012.

34. Beitz E: Aquaporin Water and solute channels from malaria parasites and other pathogenic protozoa. ChemMedChem 1: 587-592, 2006.

35. Munday JC, Eze AA, Baker N, Glover L, Clucas C, Aguinaga Andrés D, Natto MJ, Teka IA, McDonald J, Lee RS, et al: Trypanosoma brucei aquaglyceroporin 2 is a high-affinity transporter for pentamidine and melaminophenyl arsenic drugs and the main genetic determinant of resistance to these drugs. J Antimicrob Chemother 69: 651-663, 2014.

36. Faghiri $\mathrm{Z}$ and Skelly PJ: The role of tegumental aquaporin from the human parasitic worm, Schistosoma mansoni, in osmoregulation and drug uptake. FASEB J 23: 2780-2789, 2009.

37. Gourbal B, Sonuc N, Bhattacharjee H, Legare D, Sundar S, Ouellette M, Rosen BP and Mukhopadhyay R: Drug uptake and modulation of drug resistance in Leishmania by an aquaglyceroporin. J Biol Chem 279: 31010-31017, 2004. 
38. Braschi S, Curwen RS, Ashton PD, Verjovski-Almeida S and Wilson A: The tegument surface membranes of the human blood parasite Schistoma mansoni: A proteomic analysis after differential extraction. Proteomics 6: 1471-1482, 2006.

39. Faghiri Z, Camargo SM, Huggel K, Forster IC, Ndegwa D, Verrey F and Skelly PJ: The tegument of the human parasitic worm Schistosoma mansoni as an excretory organ: The surface aqp SmAQP is a lactate transporter. Plos One 5: e10451, 2010.

40. Mitani-Ueno N, Yamaji N, Zhao FJ and Ma JF: The aromatic/arginine selectivity filter of NIP aquaporins plays a critical role in substrate selectivity for silicon, boron, and arsenic. J Exper Bot 62: 4391-4398, 2011.
41. Figarella K, Uzcategui NL, Zhou Y, LeFurgey A, Ouellette M, Bhattacharjee $\mathrm{H}$ and Mukhopadhyay R: Biochemical characterization of Leishmania major aquaglyceroporin LmAQP1: Possible role in volume regulation and osmotaxis. Mol Microbiol 65: 1006-1017, 2007.

42. Mukhopadhyay R, Mandal G, Atluri VS, Figarella K, Uzcategui NL, Zhou Y, Beitz E, Ajees AA and Bhattacharjee H: The role of alanine 163 in solute permeability of Leishmania major aquaglyceroporin LmAQP1. Mol Biochem Parasitol 175: 83-90, 2011.

43. Kuwahara M, Asai T, Sato K, Shinbo I, Terada Y, Marumo F and Sasaki S: Functional characterization of a water channel of the nematode Caenorhabditis elegans. Biochim Biophys Acta 1517: $107-112,2000$ 